\title{
Decomposition of High Speed Jet Noise: Source Characteristics and Propagation Effects
}

\author{
R. H. Schlinker ${ }^{1}$, J. C. Simonich ${ }^{2}$, R. A. Reba. ${ }^{3}$ \\ United Technologies Research Center, E. Hartford, Connecticut., 06108, USA \\ Tim Colonius ${ }^{4}$ \\ California Institute of Technology, Pasadena, California, 91106, USA \\ and \\ F. Ladeinde ${ }^{5}$ \\ TTC Technologies, Inc., Stony Brook, New York, 11790, USA
}

\begin{abstract}
Current research programs directed at supersonic engine exhaust noise reduction are demonstrating benefits of 3-4 dBA using passive methods to increase jet mixing and break up shock cells in over-expanded flows. While progress is being made, high speed jet noise continues to be a research challenge for small business jets and tactical military aircraft. The current work benchmarks high speed jet noise using laboratory scale jets for the purpose of a) identifying source and propagation mechanisms, and b) providing validation data for simulation/modeling methods. Laboratory scale experiments are presented over a Mach number range of $M=0.68$ to 1.5 with static temperature ratio ranging from $\mathrm{Tr}=0.68$ to 2. A unique near field rotating phased microphone array technique was used to identify the large-scale turbulence structure noise source and Mach waves in supersonic shock-free jets. A companion paper documents the near field pressure statistics and projection of the convected wave packet to the far field. Validation against the directly measured far field levels quantitatively establishes the large scale structure noise contributions. The combined studies underpin a long term effort to develop modeling methods and new concepts for jet noise suppression based on controlling the evolution of the large-scale turbulence structures.
\end{abstract}

\section{Introduction}

\section{A. Problem Statement and Long Term Goal}

Current research programs directed at supersonic engine exhaust noise reduction are demonstrating benefits of 3-4 dBA using passive methods to increase jet mixing and break up shock cells in over-expanded flows. Approaches include mechanical chevrons, fluid/air injection used as deployable chevrons, and lobed nozzle inserts to achieve ideal expansion. Arguably, achieving dramatic improvements in noise reduction will require a multifaceted approach combining unique engine/airframe architectures, and new component-level technologies. In the long term, our research effort aims to develop component-level technologies based on controlling instability waves/large-scale structures known to be the source of aft-angle noise. Noise control strategies are envisioned based on modifying the instability wave evolution, breakdown, and spatio-temporal structure by introducing unsteady forcing in the initial shear layer region. Since this region is highly receptive to perturbations, the actuator authority and power requirements will be significantly lower compared to requirements for steady forcing reported in prior studies. While this concept has been envisioned for many years, its development and impact on noise reduction has

\footnotetext{
${ }^{1}$ Project Leader, Thermal Fluid Sciences Department, 411 Silver Lane, MS 129-17, AIAA Associate Fellow

${ }^{2}$ Staff Engineer, Thermal Fluid Sciences Department, 411 Silver Lane, MS 129-17, AIAA Senior Member

${ }^{3}$ Project Leader, Thermal Fluid Sciences Department, 411 Silver Lane, MS 129-17, AIAA Member

${ }^{4}$ Professor, Department of Mechanical Engineering, AIAA Senior Member

${ }^{5}$ Director of Research, P. O. Box 1527, AIAA Life Member and Associate Fellow
} 
been hindered by the lack of physics-based control strategies. Prior studies focused primarily on actuator devices for mixing enhancement. Therefore, our efforts include development of unique modeling and experimental diagnostic capabilities suited for use in identifying control strategies.

The current study is one element of a comprehensive program to: 1) develop the fundamental knowledge of cause-and-effect between large-scale structures and far field noise, and 2) develop validation data for models/simulation methods for noise generation by large-scale turbulent structures with the long term goal of developing control strategies. With this objective in mind UTRC, Caltech (Professor Tim Colonius), and TTC Technologies (Dr. Foluso Ladeinde) organized a Team to develop computational models and software for predicting large scale structure turbulence noise in high-speed jets, with fidelity and computational expense suited for engineering analysis. The capability of TTC's Reynolds-averaged Navier Stokes (RANS) and unsteady Large Eddy Simulation (LES) based simulations is being assessed with a focus on the ability to capture, with sufficient accuracy and affordable computational cost, the relevant features of large-scale turbulence noise sources controlling the low frequency spectral peak at aft angles. In addition, RANS based models utilizing stability methods are being developed at Caltech as a computationally inexpensive reduced-order method.

\section{B. UTRC Role}

In the context of this larger goal, UTRC's role has been to develop the fundamental diagnostic methods to detect the turbulent flow structures being modeled, and to create an extensive experimental validation data base for the LES and RANS-based prediction methods. The current program also focuses on quantitatively projecting the flow structure of the near field pressure statistics to the far field to demonstrate cause-effect of the source mechanism and determine the source contribution. This was motivated by the turbulence structure noise mechanism being cited as a strong contributor among the aeroacoustic technical community although quantitative confirmation has been limited.

The overall program requirements led to development of a novel near field phased array to measure the organized structure turbulence unsteady pressure statistics at the critical shear layer interface between the jet flow and the acoustic radiation field (Figure 1). This region, referred to as the hydrodynamic near field, is viewed as the sound "source" containing the traveling wave pressure signature responsible for noise radiating to the far field in the aft direction. The source field can be measured with microphones just outside the non-linear turbulent flow region for comparison with the LES and the instability predictions. Spectral features and length scales of the large-scale turbulence noise sources are measured directly and used to develop a convective wave packet model to link the near field and the far field.
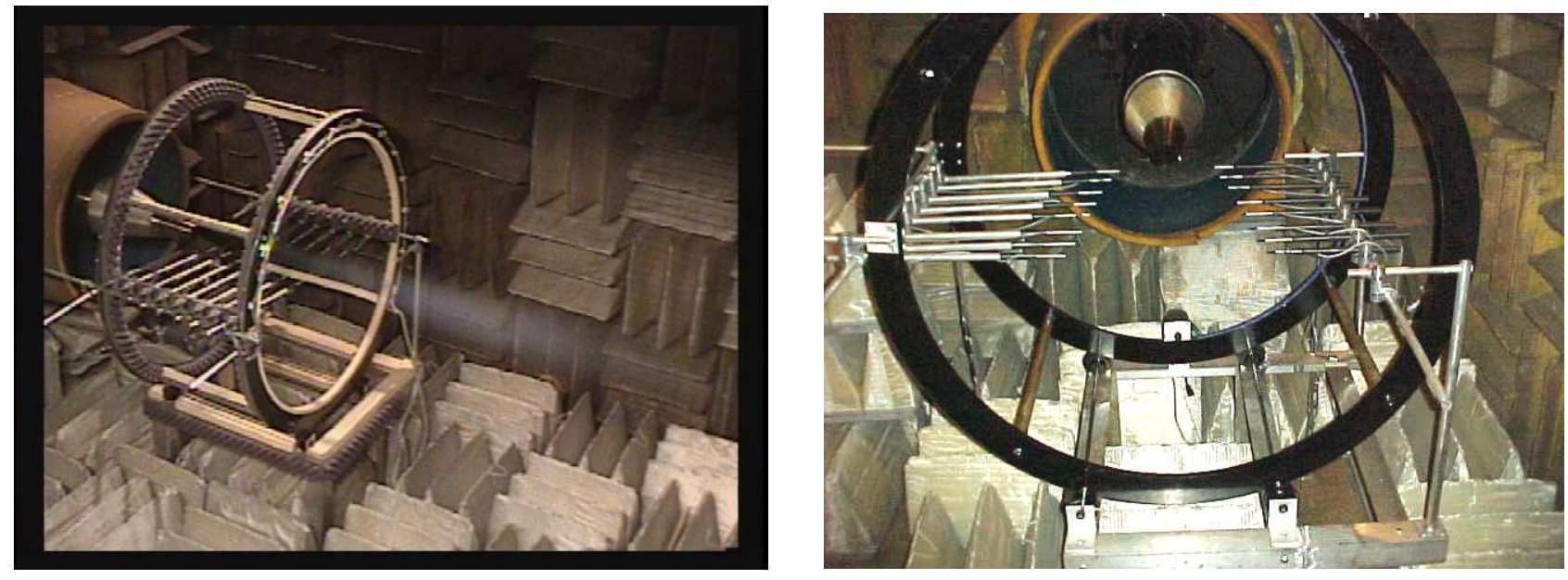

a)

b)

Figure 1. a) Rotating array installed in UTRC open jet Acoustic Research Tunnel for supersonic jet noise study; b) View looking upstream into open jet with rotating array in plane of reference array. 
UTRC also functions as the assessment site, providing blind prediction versus experiment comparisons spanning high subsonic to supersonic exhaust velocities. Such assessments also include examination of fundamental physics of large scale structure noise generation to guide improvements to the models. To facilitate progress in this fundamental area, the laboratory scale supersonic nozzles used in the current study have been designed for shockfree operation. To check relevance to full scale engine noise, comparisons are made with prior studies conducted by UTRC on supersonic exhaust noise as for example reported by Schlinker, Liljenberg, Polak, Post, Chipman, and Stern in Reference 1.

\section{Prior Near Field-to-Far Field Studies}

Since one element of the current paper is on the diagnostic technique identifying the organized structure noise and the associated jet noise data base, a brief background is provided on the experimental approaches used over the years to infer organized structure noise. Some of the earliest studies of pressure fluctuations due to coherent structures and their linkage to the far field spectra were conducted by Mollo-Christensen (Ref. 2). Other investigators measured inflow density and velocity fluctuations simultaneously with the far field noise (Ref. 3-6) to determine causality. Analytical investigations of the jet spatial coherence responsible for jet noise generation were conducted by Michalke (Ref. 7) motivated by the experiments of Fuchs (Ref. 8). Panda, Seashotlz, and Elam (Ref. 9) linked the density fluctuations with far field acoustic signals followed by Ukeiley and Ponton (Ref. 10) using nonintrusive methods to measure the three dimensional character of the hydrodynamic pressure fluctuations over a large axial extent of the jet. The latter study postulated that the far field noise is controlled by low azimuthal mode numbers.

Suzuki and Colonius (Ref. 11) developed a phased array technique to detect instability waves in the hydrodynamic near field in a study conducted in partnership with James Bridges at NASA GRC. They demonstrated analytically that pressures are consistent with instability waves evolving in the turbulent shear layer mean flow up to the end of the potential core. More general eduction methods have been developed by Tinney, Jordan, Antoine, and Delville (Ref. 12) and Muller, Vuillot, Rahier, Casalis, and Piot (Ref. 13) to identify pressure characteristics associated with noise generation. Recent studies by Hileman et al (Ref. 14, 15) have coupled microphone measurements with high speed flow visualization to provide insights into the evolution of flow structures and the coupling with noise generation and radiation to the far field.

Relative to controlling the turbulent shear layer flow evolution, there have been many prior forced jet experiments focused on actuator technology development and understanding the dynamic response of the jet. These studies did not include modeling/simulation of the flow physics for noise control so that noise often increased rather than being decreased. Recent experimental studies by Samimy, Kim, Kastner, and Adamovich (Ref. 16) have, however, provided a few $\mathrm{dB}$ of noise reduction at high Strouhal number using localized plasma actuators arranged in a sophisticated architecture. These results provide promise for the future.

While the above near field studies provided significant progress towards understanding noise sources, the reported measurements were not used to directly connect the near field and the far field via a simultaneous sourceto-far field projection method. Progress towards such a projection was made by Reba, Narayanan, Colonius, and Suzuki in Reference 17 utilizing the data of Suzuki and Colonius. Although the study was limited to subsonic jet noise, it provided the first link between near field array data and far field spectra. The Team effort between UTRC and Caltech improves the diagnostic method using a different phased geometry and extends the technique to supersonic jet flows.

\section{Other Published Results by the Team}

An overview of the broader program is summarized here beginning with the companion paper by Reba, Simonich, and Schlinker (Ref. 18). This study develops the projection method used to translate the measured near field pressure statistics to the far field via the linear wave equation, and demonstrates that a simple Gaussian wavepacket model can be used to provide a parametric model for the source characteristics. The good agreement with far field noise data indicates that the key features of the large scale organized structure acoustic source have been identified in the measurement technique. Also, the quantitative results show how the organized structure modal content contributes significantly to the power spectral density (PSD) peak at $\mathrm{St}=0.4$ in the aft direction. Much of the work is still in progress so the current paper and companion paper are interim results.

The Team also reported comparisons between LES and measured near field unsteady pressures and far field acoustic pressures based on work by Ladeinde, Cai, Alabi, Reba, Schlinker, and Simonich in Reference 19. Results are summarized here to provide an overview on the other elements of the combined Team research thrust. Overall, the LES simulations capture the spatio-temporal evolution of large-scale turbulence with reasonable accuracy. 
However, the LES-predicted near field turbulence structures appear to be significantly more energetic. These observations are generally consistent with results of other recent investigators such as Muller et al. As a result, an over-prediction of 5 and $10 \mathrm{~dB}$ is observed in the near-field pressure for the cold and hot jet simulations, respectively. For the far-field sound pressure, the LES-predicted OASPL agrees with the experimental data for the cold jet, and over predicted the hot-jet sound level by $5 \mathrm{~dB}$. It is noted that similar discrepancies have been reported by other investigators applying LES to subsonic heated jets. The near-field results for the supersonic calculations show significantly high noise levels, particularly at low frequencies. In the far field, reasonable agreement is seen at the aft-most angles, while over-predictions are seen at the more sideward angles. These differences indicate research challenges still exist for simulation and modeling methods.

Ladeinde et al. also observed that existing validations of LES predictions for high speed jet noise are primarily based on a comparison of the far-field spectra, without a corresponding focus on the near field. The results show that, in some cases, the far-field predictions agree better with the experiments than do the near-field. Thus, comparisons on the basis of near-field source characteristics offer a more discriminating metric. Another observation pertains to the low emphasis given to the errors from simulations, even for the far-field results. In this case, some of the errors observed in calculations reported by others are actually of larger magnitudes that the target noise reduction levels, suggesting a need to improve the accuracy of those calculations. Detailed analysis and resolution of the sources of discrepancy are currently in progress.

The Caltech team is pursuing a combined RANS-based prediction of the mean turbulent flow field with linear and nonlinear stability analysis. Models are based on a description of large-scale structures as instability waves (or wave packets) to predict near-field hydrodynamic pressure fluctuations. The approach is aimed at rapid prediction tools for far-field sound based on a lower-fidelity description of the acoustic sources than is obtained with the more computationally intensive LES. The near field wave-packet amplitudes may, in turn, through a wave equation projection approach (Ref. 17), be used to predict far-field sound associated with large scale structures.

Caltech's previous work (supported by the Aeroacoustics Research Consortium, AARC) includes experimental diagnostics on a 78 microphone near-field array fabricated at NASA Glenn Research Center (Fig. 4). Results show that up to the end of the potential core, pressure fluctuations just outside the jet shear layer are very well modeled as linear instabilities evolving in the measured (or predicted) turbulent mean flow field. The analysis has been performed by Suzuki and Colonius (Ref. 11) for circular nozzles over a range of Mach numbers and temperature ratios, and more recently, for a chevron nozzle at a single set point as reported by Gudmundsson and Colonius (Ref. 20). Nonlinear effects appear to be important further downstream, and the Caltech group is presently pursuing a nonlinear Parabolized Stability Equation (PSE) approach in order to predict wave-packet amplitudes further downstream.

\section{Approach and Experimental Details}

\section{A. Approach}

In the present study, sensing of large scale structures and development of data bases for validation of noise prediction methodology is being pursued via the following sequence of objectives:

$>$ develop a fundamental experimental validation approach and diagnostic methods to acquire a unique near field data base confirming the large scale structures

$>$ acquire model scale noise and flow field data to assess the LES and RANS based instability models in addition to validating a projection method

$>$ conduct the comparisons between blind near field and far field prediction cases and the model scale measurements

$>$ establish the source mechanisms

$>$ recommend prediction method improvements with the long term objective of controlling organized structure noise

As noted earlier, rather than using supersonic far field acoustic data for the organized structure prediction methodology validation, the diagnostic method in Figure 1a was developed at the critical shear layer interface between the jet flow and the acoustic radiation field. The current program utilizes this technique under the challenging conditions of supersonic flow with microphones located within two diameters of the jet centerline as show in Figure 1b. 
Data was acquired simultaneously from both near field and far field microphone arrays and stored on a digital data system as time series information to allow correlation of signals for source identification and analysis. At the same time, source to far field coupling was examined. The outcome of the current study is a decomposition of the measured acoustic data to:

$>$ characterize the far field and near field spectra and directivity as a function of Mach number and temperature ratio

$>$ determine the importance of non-linear propagation for Mach wave radiation

$>$ correlate far field microphone signals to localize the origin of the large scale structure noise and Mach wave radiation (in progress)

$>$ correlate near field microphone signals with far field signals to establish the hydrodynamic source-toacoustic radiation coupling characteristics (in progress)

\section{B. UTRC Acoustic Research Tunnel}

Experimental studies were conducted in the UTRC Acoustic Research Tunnel (ART) developed in 1970 as the first forward flight anechoic simulation facility for jet noise, fan and propeller noise, and lifting surface/airframe noise studies. The facility provides up to a 50" open jet forward flight simulation for jet noise using large single stream exhaust nozzles up to 6" diameter to achieve relevant Reynolds numbers, stagnation temperatures up to 1800 deg R, and Mach numbers (M) up to 2.5 based on the 400 psi supply air (Fig. 2). The exhaust nozzles project beyond the open jet as shown in Figure 3 as viewed from a linear phased array set of microphones stationed at aft angles to measure axial source distributions. Boundary layer suction exists on the model nozzle exterior to control boundary layer thickness for simulation of full-scale engine nacelle external flows. The free field microphones (Fig. 3) located outside the $\mathrm{M}=0.1$ to 0.36 open jet flow provide sound pressure level measurements over the key directivity angles ranging from $80 \mathrm{deg}$ to $155 \mathrm{deg}$ from the engine inlet centerline. Higher Mach numbers up to $\mathrm{M}=0.6$ are available with a 21 " 331 " open jet test section. The study reported here did not apply the linear array in Figure 3, but, used the rotating array in Figure 1. Forward flight simulation was not used since the objective was to first gain a fundamental understanding of large scale structure noise.

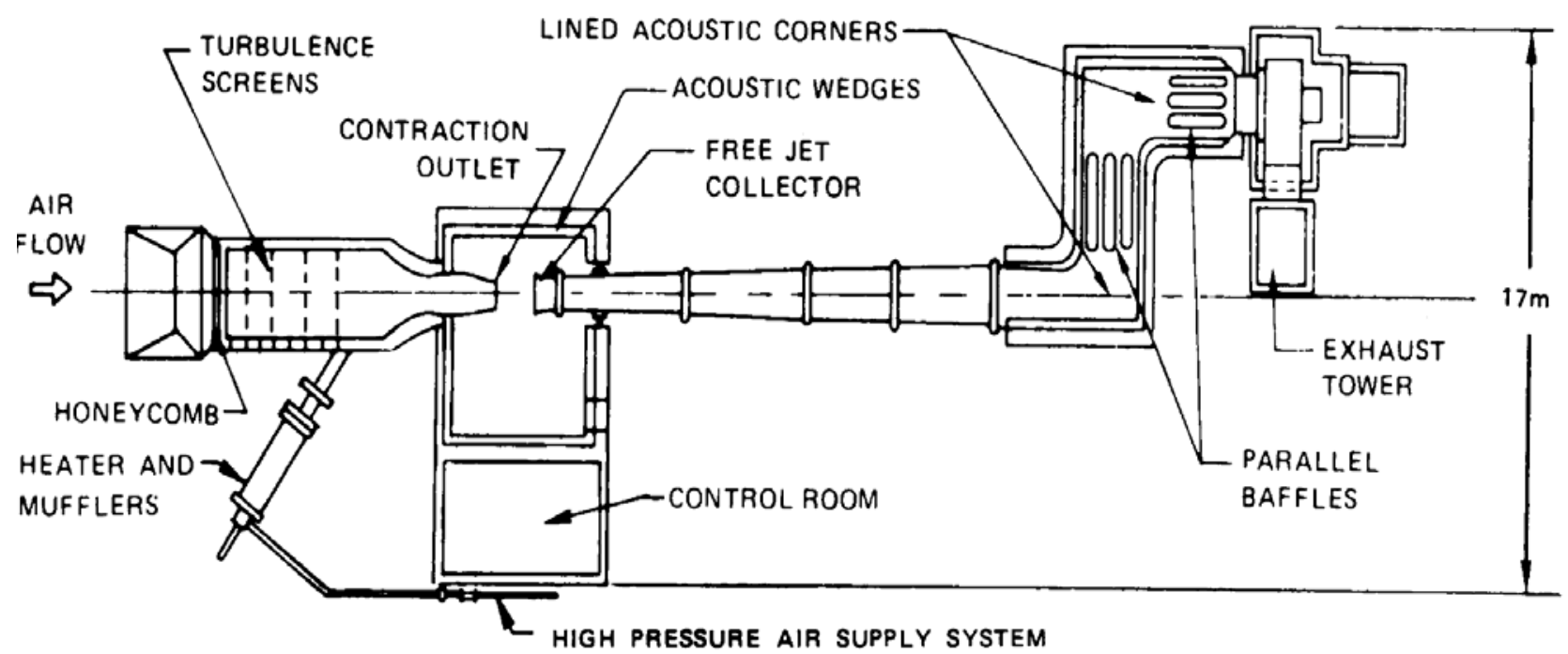

Figure 2. UTRC Acoustic Research Tunnel. 


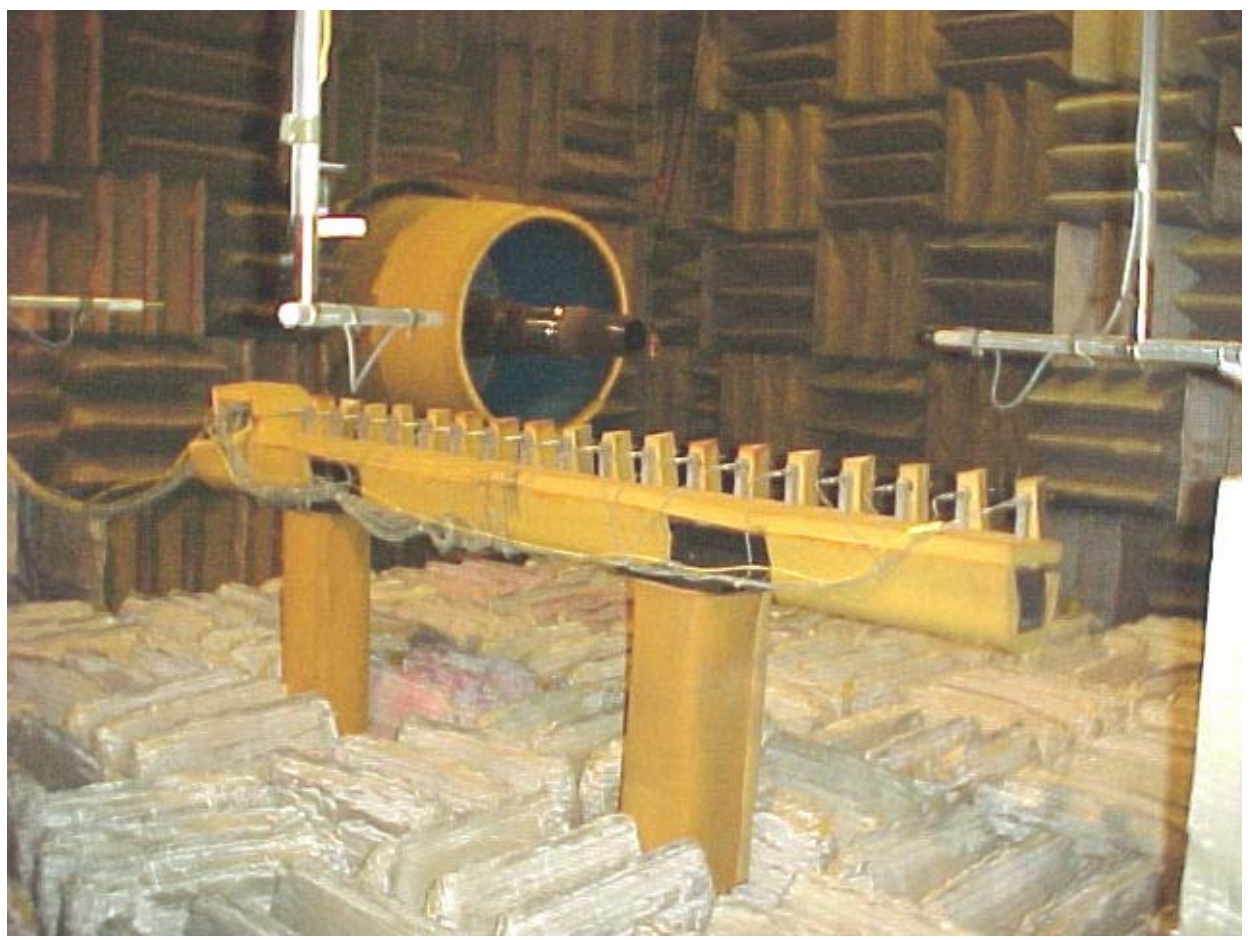

Figure 3. Acoustic Research Tunnel showing model scale nozzle as viewed from aft microphone angle.

A recent description of the facility is given in Simonich, Narayanan and Schlinker (Ref. 21) as part of a facility and data quality paper reporting UTRC "lessons learned" in previous programs. Recent jet noise reduction successes developed in this facility include a JT8D-200 engine series hush kit based on a tandem mixer/chevron concept as reported in a patent by Schlinker et al: Jet Exhaust Noise Reduction System and Method, US Patent $7,114,323$ B2. In addition, noise reduction approaches were developed in conjunction with Pratt \& Whitney during 2004 for the PW6000 engine.

The test section is surrounded by a sealed anechoic chamber 16 feet high, $18 \mathrm{ft}$ long (in the jet centerline direction) and $22 \mathrm{ft}$ wide. The chamber walls are lined with 18 inch high fiberglass wedges which provide an anechoic acoustic environment above $175 \mathrm{~Hz}$. Downstream of the test section, the air flow enters a diffuser through a circular collector with acoustic treatment on its flow impingement surface. The diffuser is designed to operate unstalled and hence is not a major source of background noise. To avoid tunnel fan noise from propagating upstream into the anechoic chamber, a Z-shaped muffling section with two right angle bends and parallel treated baffles is located between the diffuser and the fan. The flow is exhausted to the atmosphere by a $1500 \mathrm{hp}$ centrifugal fan.

The jet is supplied with high pressure air from a compressor system capable of delivering $20 \mathrm{lb} / \mathrm{sec}$ of dry air continuously. The air is heated using a liquid propane SUE burner. Flow from the combustor passes through a muffler which attenuates upstream combustion and valve noise. The airflow rate and combustor temperature are regulated by a programmed logic controller (PLC). Airflow is precisely controlled by using two valves in parallel. The larger valve is used to set the high flow rate and the small valve is used for precision control.

\section{Rotating Phased Array}

An experimental-diagnostic method was developed, comprising a non-invasive phased microphone-array technique for measuring hydrodynamic pressure of large-scale turbulent structures in the jet near field (Figure 1). The technique combines capabilities developed in recent years for subsonic jets by Caltech (Professor Colonius and Dr. Suzuki) through collaborative research involving Caltech, UTRC, and NASA-GRC (Dr. James Bridges) and the Aero-Acoustic Research Consortium (AARC).

The array hardware consists of an open frame creating an acoustically "transparent" structure to which a rotating axial array of microphones is attached while a second axial array remains fixed in location. The axial extent of the microphone array ranges from $\mathrm{X} / \mathrm{D}=0$ to 10 has been increased for 2008 studies by lengthening the microphone support rods in the axial direction. Array microphones are located on a virtual cone within 2 diameters of the nozzle 
centerline and outside of the turbulent flow. Prior experiments employing a hydrodynamic array concept (e.g. Suzuki and Colonius) used a 78 microphone, conical, fixed cage microphone array shown in the photo of Figure 4. The system consisted of 13 rings with 6 microphones per ring, allowing decomposition of the pressure signals into the axisymmetric $(\mathrm{m}=0)$ and first two azimuthal Fourier modes $(\mathrm{m}=1,2)$.

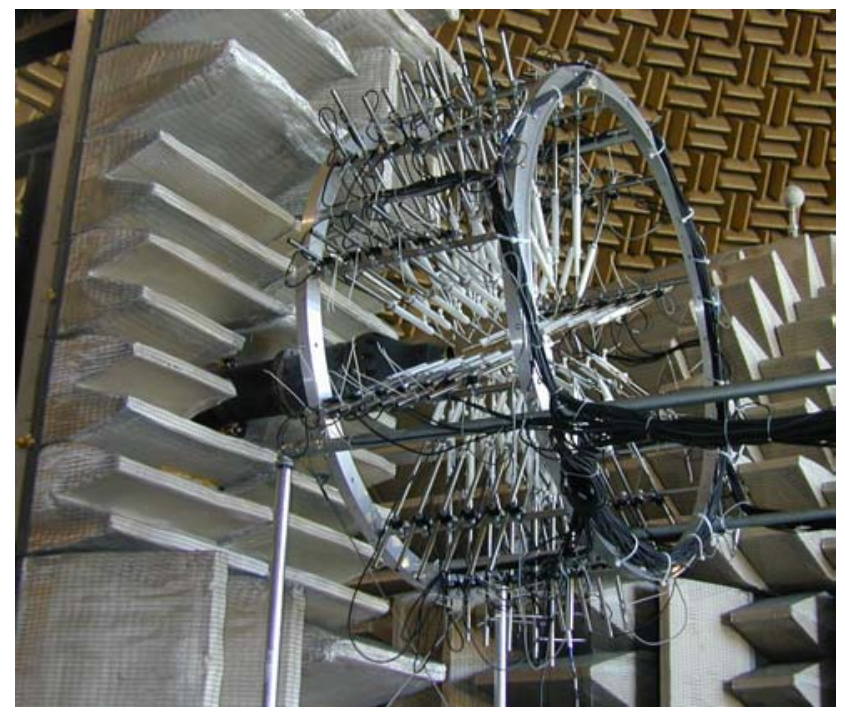

Figure 4. 78 microphone array tested in NASA GRC SHJAR for subsonic jets.

The operation of the current "dual arrays" is illustrated in Figure 5. One array rotates in the azimuthal direction, thereby enabling measurement of azimuthal correlations for any choice of reference location and azimuthal spacing. In this dual rotating concept, the modal content at any axial location is determined by Fourier transformation of the two-point azimuthal correlation. For the mechanical embodiment shown in Figure 5, one linear array is moved automatically in the azimuthal direction, and the second remains fixed for circular nozzles. The rotating feature developed here represents a significant advance beyond the current approaches requiring many fixed sensors. It also minimizes the number of microphones needed for complex geometries of interest for future noise reduction.
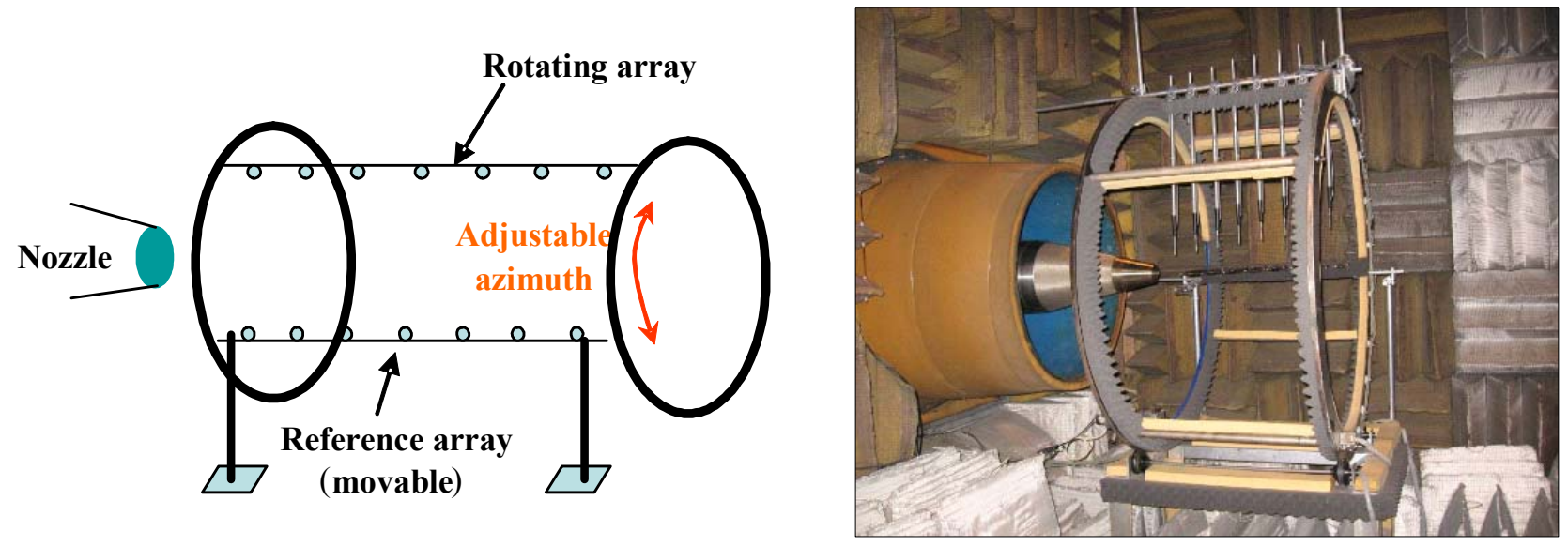

Figure 5. Rotating array operation for mapping shear layer hydrodynamic near field. 
Many jet noise concepts of practical interest (e.g. chevrons/lobes with $\mathrm{N}>6$, rectangular nozzles, and dynamic shear-layer forcing) introduce significant turbulence energy in higher mode orders, beyond those measured in the round nozzle configuration of Figure 1b. In this case, application of the hydrodynamic array to such geometries would require a significant increase of the microphone count per ring to avoid aliasing errors. This drives the geometry back to the earlier microphone array geometry with many fixed microphones which is impractical due to limitations on data acquisition systems and the high cost of quality microphones. In order to enable application of the diagnostic method to acquire validation data for noncircular nozzles, which introduces turbulence energy at higher azimuthal modes $(\mathrm{m}>2)$, the moveable reference array (Figure 5) was introduced. For a given position of the reference array, phase-locked data between the two arrays is acquired for each location of the movable azimuthal array while the number of locations is governed by the desired circumferential modal resolution. The current dual rotating array concept utilizes axial microphone spacing based on the desired axial resolution but can accommodate up to 30 microphones in each array when needed for non-circular nozzles with significant energy at higher mode orders.

\section{Nozzles, Flow Conditions, and Flow Fields}

Nozzle flow conditions for the model scale tests were selected based on typical Mach numbers and temperatures associated with small business jets and military engines. A converging nozzle was designed for subsonic operation while converging-diverging nozzles were designed for $M=1.2,1.5$ and 2.0. Shock free conditions were chosen for the supersonic cases to facilitate the detection of organized structures in the jet near field without the presence of shock cells. Future studies will pursue the interaction of organized structures with shock cells at off-design conditions. However, a long term expectation is that the mechanical design of full scale engine controls for the nozzle throat area (A8) and nozzle exit area (A9) will provide shock-free operation to avoid the additional screech and broadband shock-noise source mechanisms. Shock free operation would only be limited, then, by the imperfect expansion in the straight diffuser ramp section of the nozzle.

Static temperature ratios (Tr) in the test matrix, relative to a nominal upstream plenum temperature of $72^{\circ} \mathrm{F}$, ranged from cold (without heating) to $\operatorname{Tr}=2$ with a few test cases at $\operatorname{Tr}=2.88$. Nozzle exit diameters $\left(\mathrm{D}_{\mathrm{j}}\right)$ were held to a consistent 3" size over the range of Mach numbers.

Mean flow data was acquired using a multi-probe mounted on a diamond shaped airfoil attached to the ART multidirectional traverse system which allows three directions of translation in a Cartesian coordinate system. The traverse base consists of $12 \mathrm{ft}$ bed aligned parallel to the model scale nozzle centerline for axial surveys. Transverse surveys are conducted with two additional independent slides mounted on the axial bed. The multiple directions are remotely controlled via the ART computerized data acquisition system.

Due to the thermal growth of the piping system at high temperatures a procedure to locate the jet centerline was developed. This is especially critical for the far downstream locations when acquiring flow field and near field data. Preliminary horizontal and vertical traverses shown in Figure 6 were used to determine the common slopes of pressure versus radial distance on both sides of the jet from which the jet centerline was then determined using interpolation.

\section{E. Instrumentation and Data Systems}

Nozzle flow controls and electronic instrumentation were set up in the ART Control Room shown in Figure 7. B\&K type 4535 and $49391 / 4$ " microphones were used for the near field phased array system and for the far field stations respectively. Phased array microphone signals were amplified using Nexus amplifier clusters. Precision Filters Corporation filters were used in the control room for signal conditioning. A DataMAX II 40 channel transportable data acquisition unit with a $40 \mathrm{G}$ hard drive was used for data storage and post processing. Calibration of the near field and far field microphone systems for amplitude and, in particular phase, was conducted using a calibrator system developed by B\&K specifically for UTRC. 

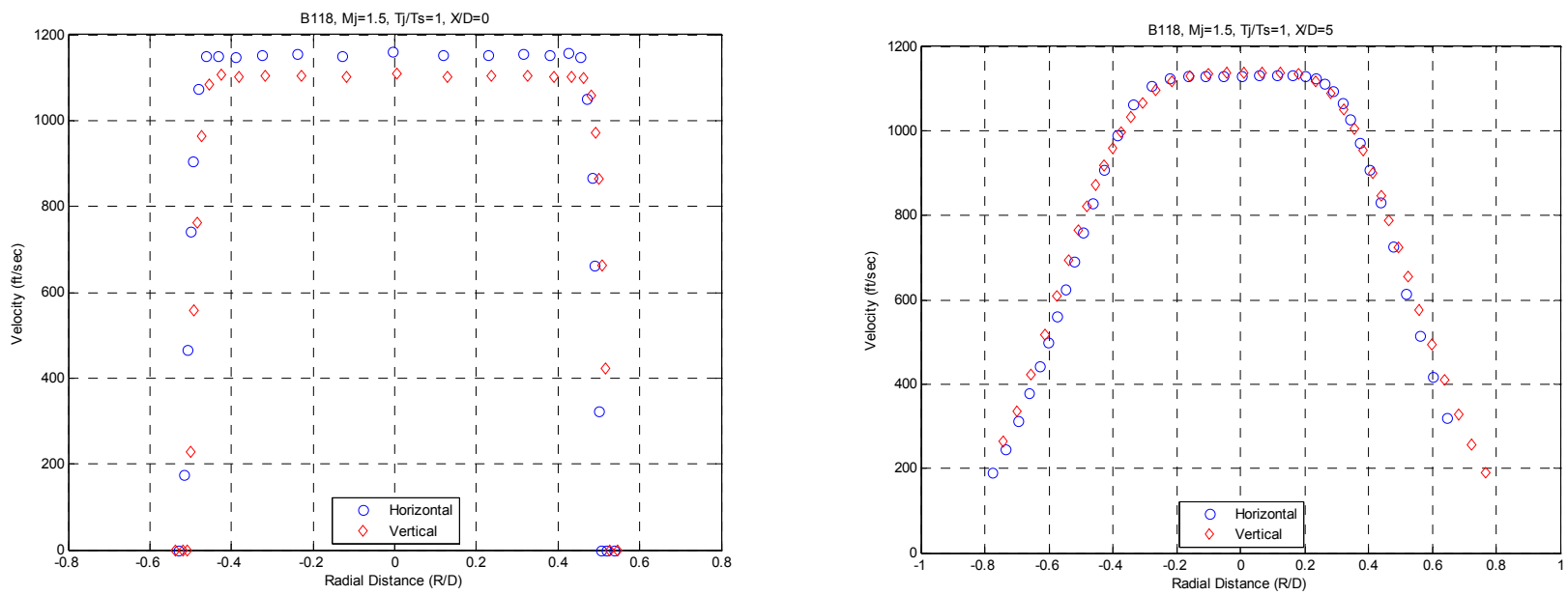
a) $X / D=0$
b) $\mathrm{X} / \mathrm{D}=\mathbf{5}$

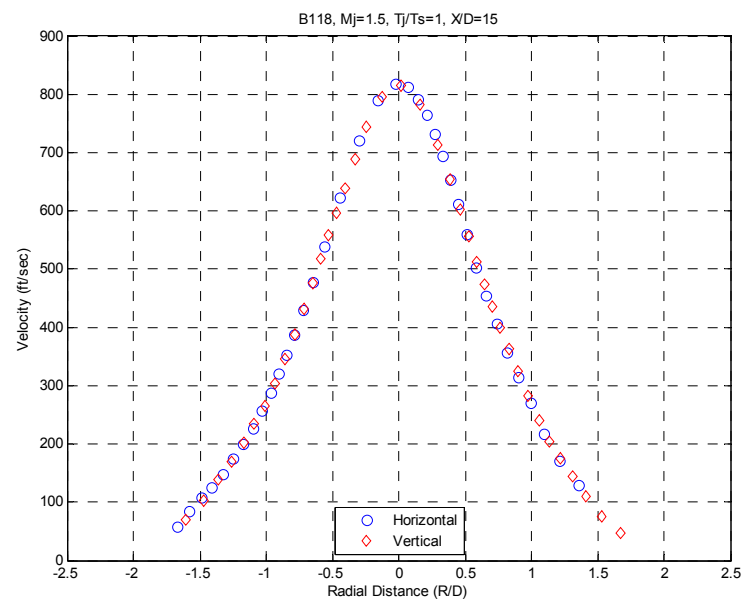

c) $\mathrm{X} / \mathrm{D}=15$

Figure 6. Typical radial velocity profiles for $M=1.5$ at selected $X / D ; T r=1$.

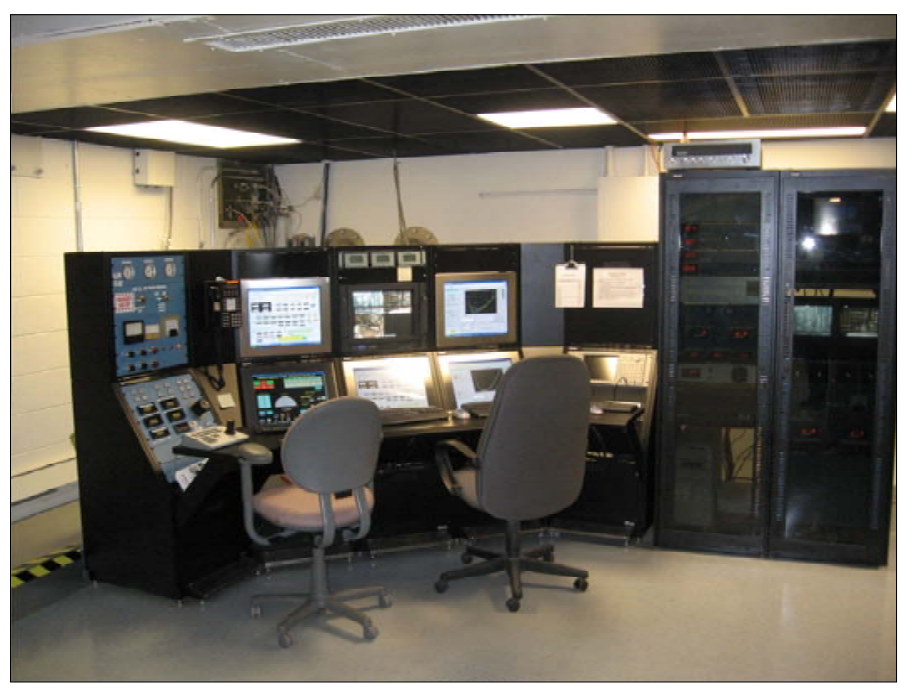

Figure 7. Acoustic Research Tunnel Control Room.

American Institute of Aeronautics and Astronautics 092407 


\section{F. Test Program and Test Matrix}

The range of data acquired to date is shown in Figure 8 for the acoustic measurements. Subsonic data at Mach number $\mathrm{M}=0.9$ was acquired for comparison with the benchmark data of Suzuki and Colonius to provide a near field data quality check over a range of temperatures ratios. An $M=1.0$ test point was used to check noise and aerodynamic characteristics at transonic conditions. The $\mathrm{M}=1.5$ test condition was investigated to simulate high speed operating conditions more typical of military engines. Other Mach numbers conditions, $M=1.2$ and $M=2$, will be tested once the already acquired data has fully interpreted. The intent is to first develop and validate the data acquisition and analysis sequence leading from experimental turbulence organized structure measurements to comparisons with spectral and length scale predictions from the simulation tools before proceeding with additional test cases. Stand alone far field acoustic data was also acquired at $\mathrm{M}=0.68$ to provide a broader range of acoustic data for tracking far field signatures such as non-linear effects.

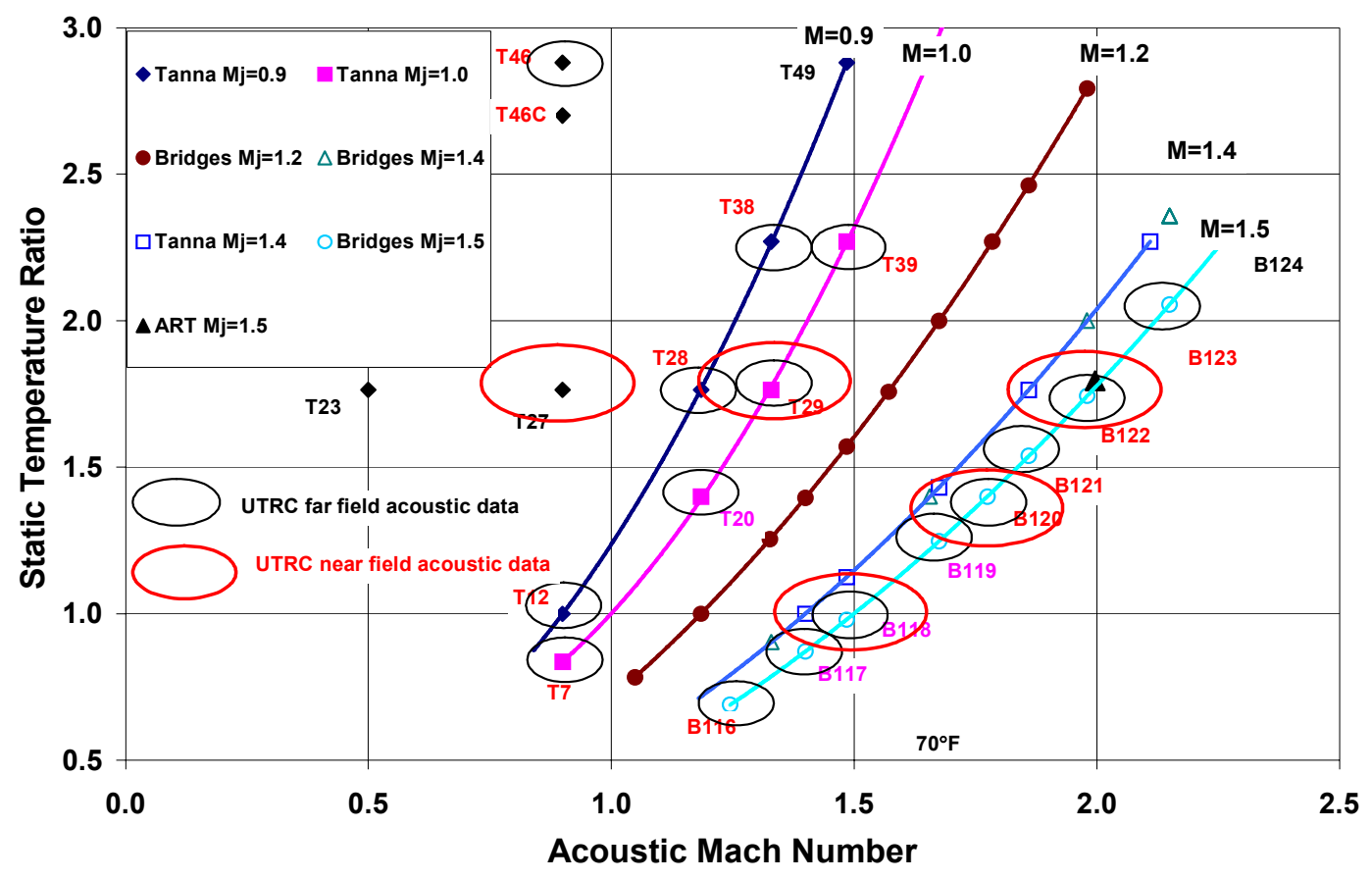

Figure 8. Operating conditions for acoustic data base linking near field sources and far field noise.

The Tanna operating conditions (Refs. 22, 23) were selected as set points for the current study since they are one of the most highly regarded and referenced heated jet noise data sets in the literature. By comparing the current data against this benchmark data set, the current measurements could be assessed. One of the set points (SP 46) was also used by Suzuki and Colonius in their earlier experiment which measured near field acoustics and could be used for comparison. The Brown and Bridges set points (Ref. 24) were chosen for the supersonic operating conditions since both aerodynamic and acoustic far field measurement data was available.

\section{A. Data Quality Check}

\section{Far Field Acoustic Results}

The quality of the far field data is confirmed in Figure 9 which shows good agreement with acoustic spectra of Bridges and Tanna at aft angles where organized structure noise dominates. Atmospheric corrections have been applied to the data accounting for temperature and humidity effects based on the approach developed by Bass, Sutherland, Zuckerman, Blackstock, and D.M. Hester (Ref. 25). 

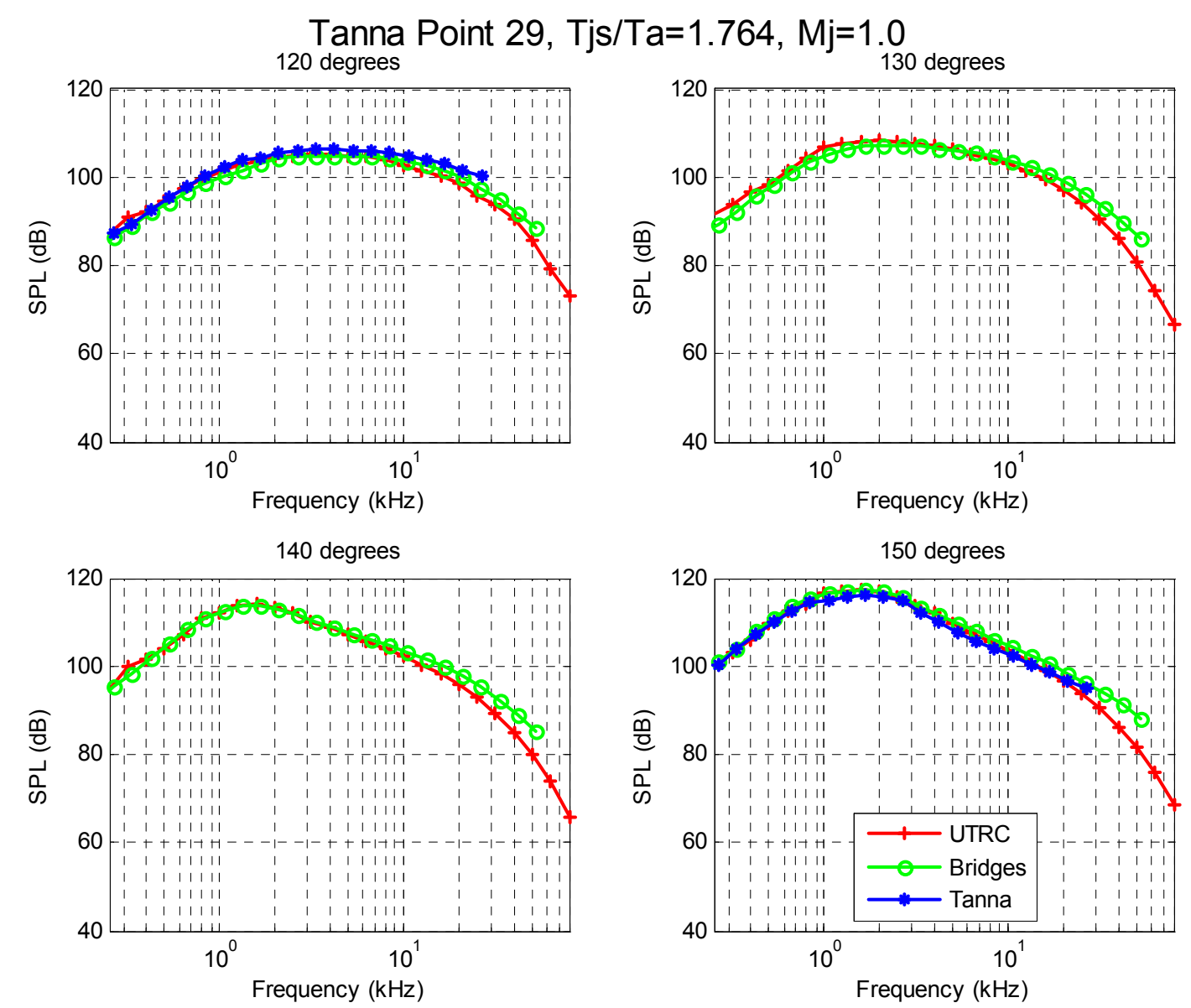

Figure 9. Far field acoustic measurements at aft angles compared with prior studies of Tanna (Refs. 22, 23) and Brown \& Bridges (Ref. 24).

\section{B. Sound Pressure Level Dependence on Directivity Angle and Strouhal Number}

Figure 10 shows normalized contours of sound pressure level as a function of directivity angle, theta, and Strouhal number, St, for the supersonic model scale test condition $\mathrm{M}=1.5$. Here theta corresponds to angles measured from the inlet axis while St number is derived from $1 / 3$ band data processing. Static temperature ratio varies from $\mathrm{Tr}=0.689$ (top left corner) to 2.055 (bottom right corner) corresponding to a velocity ratio of 1.8 over this range. Deltas between contour lines correspond to $2 \mathrm{~dB}$. The data has been corrected to standard conditions (77 $\mathrm{F}$ and $70 \%$ relative humidity).

Peak frequencies in Figure 10 occur in the $\mathrm{St}=0.2-0.4$ range in agreement with subsonic conditions and classical jet noise characteristics. As temperature/velocity increases, the spectral peak shifts forward from theta $\sim 150$ deg to $\sim 135$ deg. while the overall spectrum shape broadens.

To compare the current and prior model scale studies with full scale engine data, Figure 11a shows normalized contours from the full scale engine supersonic test reported in Reference 1. (Note that Mach number values could not be published for this engine). Similar to Figure 10 the spectral peak in Figure 11a occurs at 135 deg. The additional acoustic radiation in the forward direction, concentrated in the narrow range of St $\sim 0.4-0.8$, was due to broadband shock noise in the engine data.

For comparison with subsonic jet noise, the $\mathrm{M}=0.9$ condition in Figure $11 \mathrm{~b}$ indicates that the spectral peak occurs at theta $\sim 150$ degrees similar to the lowest temperature ratio supersonic Mach number condition in Figure 10. Contours in this subsonic case are derived from ARP 876 (Ref. 26) for a jet with the same exit diameter as the engine data in Figure 11a. 


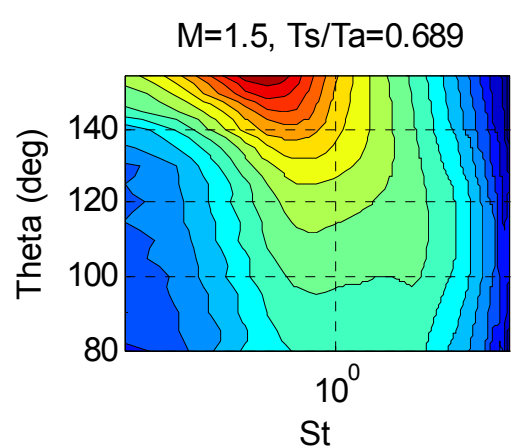

St

$\mathrm{M}=1.5, \mathrm{Ts} / \mathrm{Ta}=1.247$

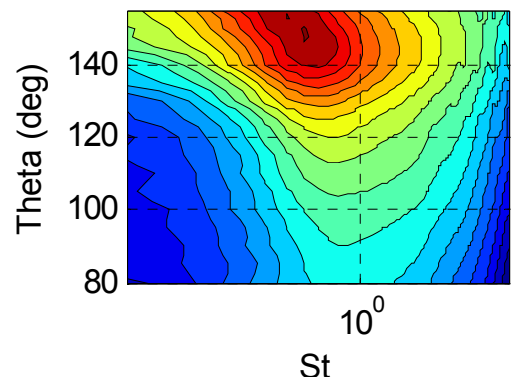

$M=1.5, T s / T a=1.743$

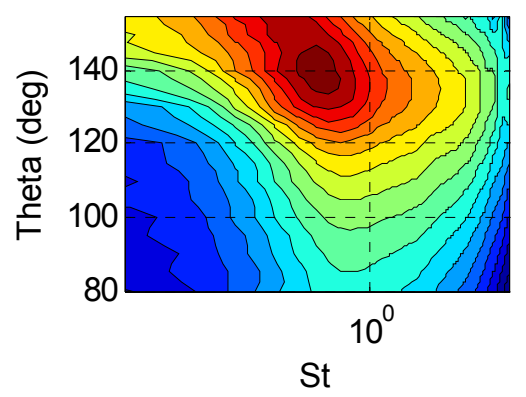

$\mathrm{M}=1.5, \mathrm{Ts} / \mathrm{Ta}=0.871$

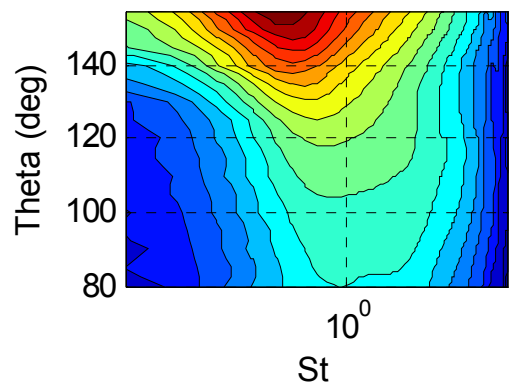

$\mathrm{M}=1.5, \mathrm{Ts} / \mathrm{Ta}=1.4$

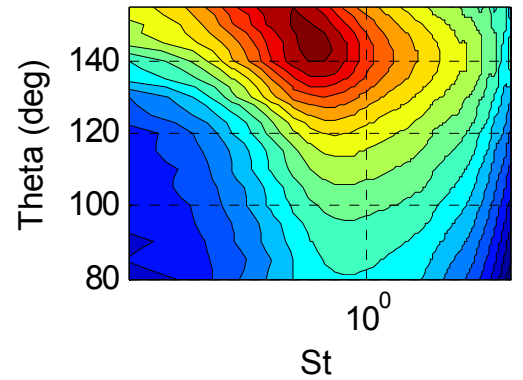

$M=1.5, \mathrm{Ts} / \mathrm{Ta}=2.055$

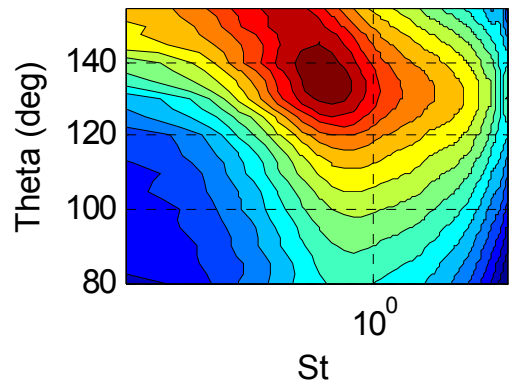

$\mathrm{M}=1.5, \mathrm{Ts} / \mathrm{Ta}=0.98$

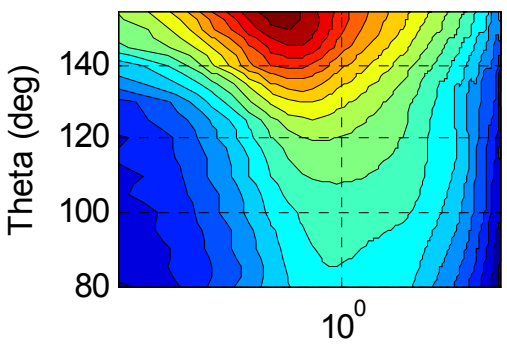

St

$M=1.5, T s / T a=1.538$

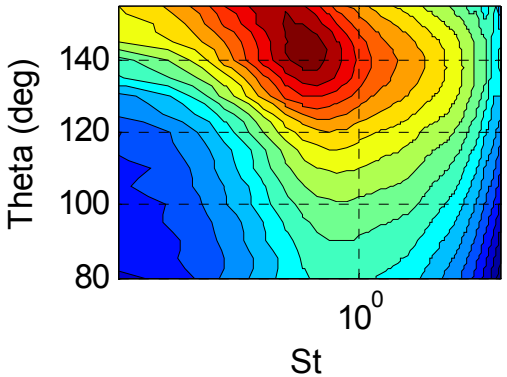

St

Figure 10. Jet noise directivity as a function of static temperature ratio Ts/Ta; $M=1.5$; $2 \mathrm{~dB}$ deltas between contour lines.

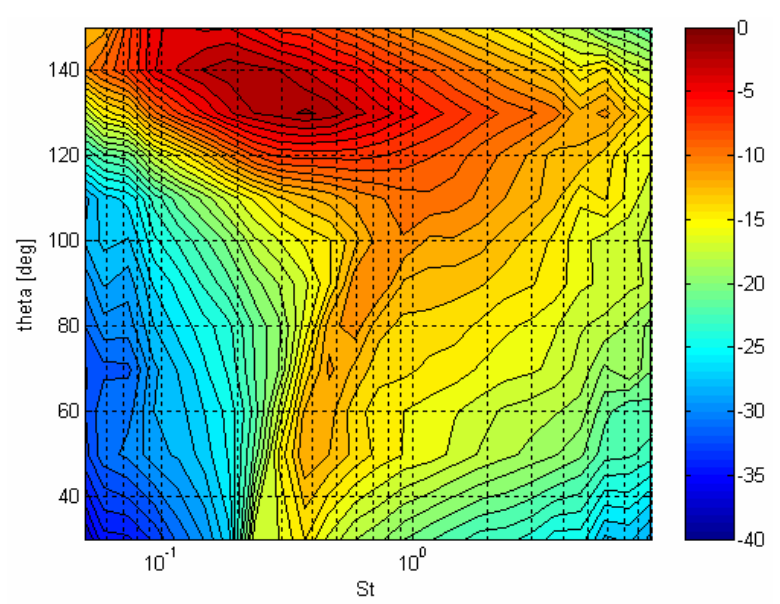

a)

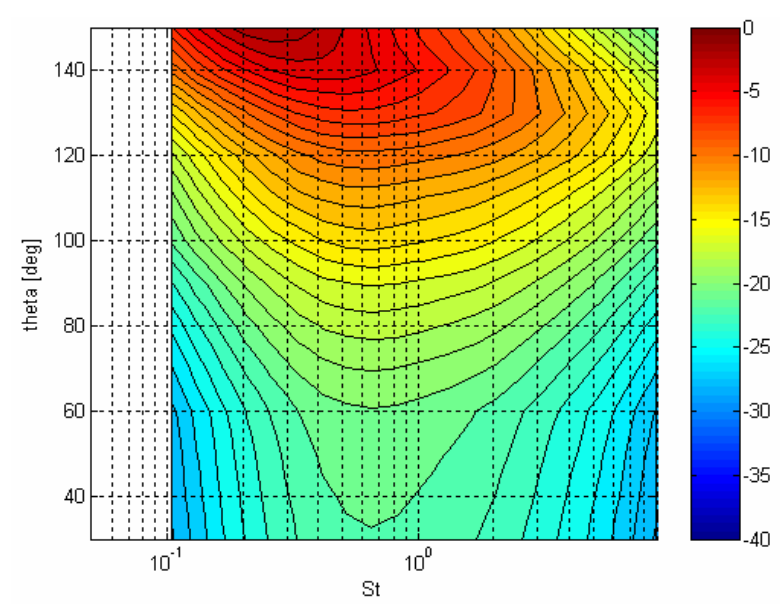

b)

Figure 11. Jet noise directivity comparison at sideline stations: a) Supersonic exhaust engine at high set point; b) Subsonic exhaust jet at $\mathrm{Mn}=\mathbf{0 . 9}$. 
In summary, the present supersonic model scale and the recently published engine scale (Ref. 1) directivity patterns display several distinct features: 1) a "beam" of acoustic energy which is narrower than subsonic radiation patterns and approximately $20 \mathrm{deg}$. forward of the subsonic directivity peak, 2) a broader spectrum at the peak directivity angle compared to subsonic conditions, and 3) rapid sound pressure level changes with directivity angle for fixed frequencies. These similar features indicate that the model scale study is capable of simulating the engine conditions.

\section{OASPL Velocity Dependence on Jet Exhaust Velocity}

Figure 12 shows OASPL levels as a function of directivity angle as jet exit velocity increased for the $\mathrm{M}=1.5$, $\operatorname{Tr}=1.76$ nozzle due to heat addition. Plotted for comparison is a $\mathrm{V}^{8}$ velocity dependence (red line in plots). As reported in the literature, and recently consolidated by Tam et al. (Ref. 27), OASPL at forward radiation angles varies with a velocity exponent $\mathrm{n}<8$ as is evident in Figure 12. At aft angles, $\mathrm{n}>8$. However, an additional feature obtained by correlating the data in the present format is that extreme aft radiation angles display an invariant OASPL with increasing velocity/temperature. This feature is evident in the "flat" plateau annotated in the 150 and 155 deg plots in Figure 12. Onset of this feature, marked by the notation "Break point" in the figure, progressively shifts to lower velocities at larger directivity angles. The invariance is accentuated at further aft angles in recent raw data provided by James Bridges (from Brown and Bridges (Ref. 24)) for the same conditions and plotted in the same format in Figure 13. In this case, data is presented for the furthest aft microphone stations in the NASA GRC dome facility.
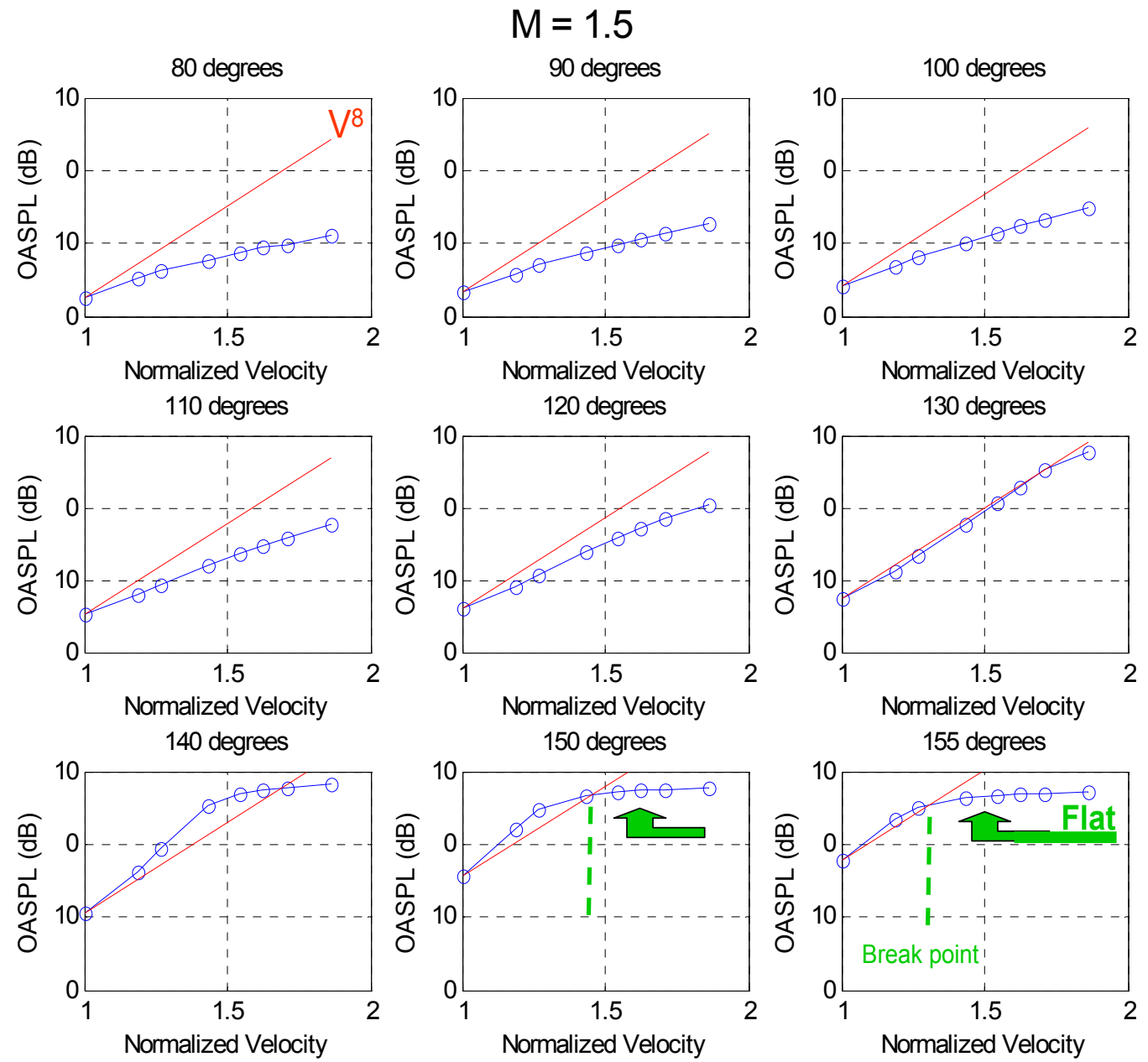

Figure 12. Overall sound pressure level versus jet velocity for range of directivity angles: $M=1.5, \operatorname{Tr}=1.76$. 
A similar invariance was observed for the full scale engine data reported earlier by Schlinker et al. (Ref. 1). At the highest temperature in the set points for the engine, the velocity scaling law transitioned to $a \sim V^{2}$ dependence. In this case, the noise increased by $\sim 3 \mathrm{~dB}$ rather than $9 \mathrm{~dB}$ projected from $\mathrm{V}^{8}$.
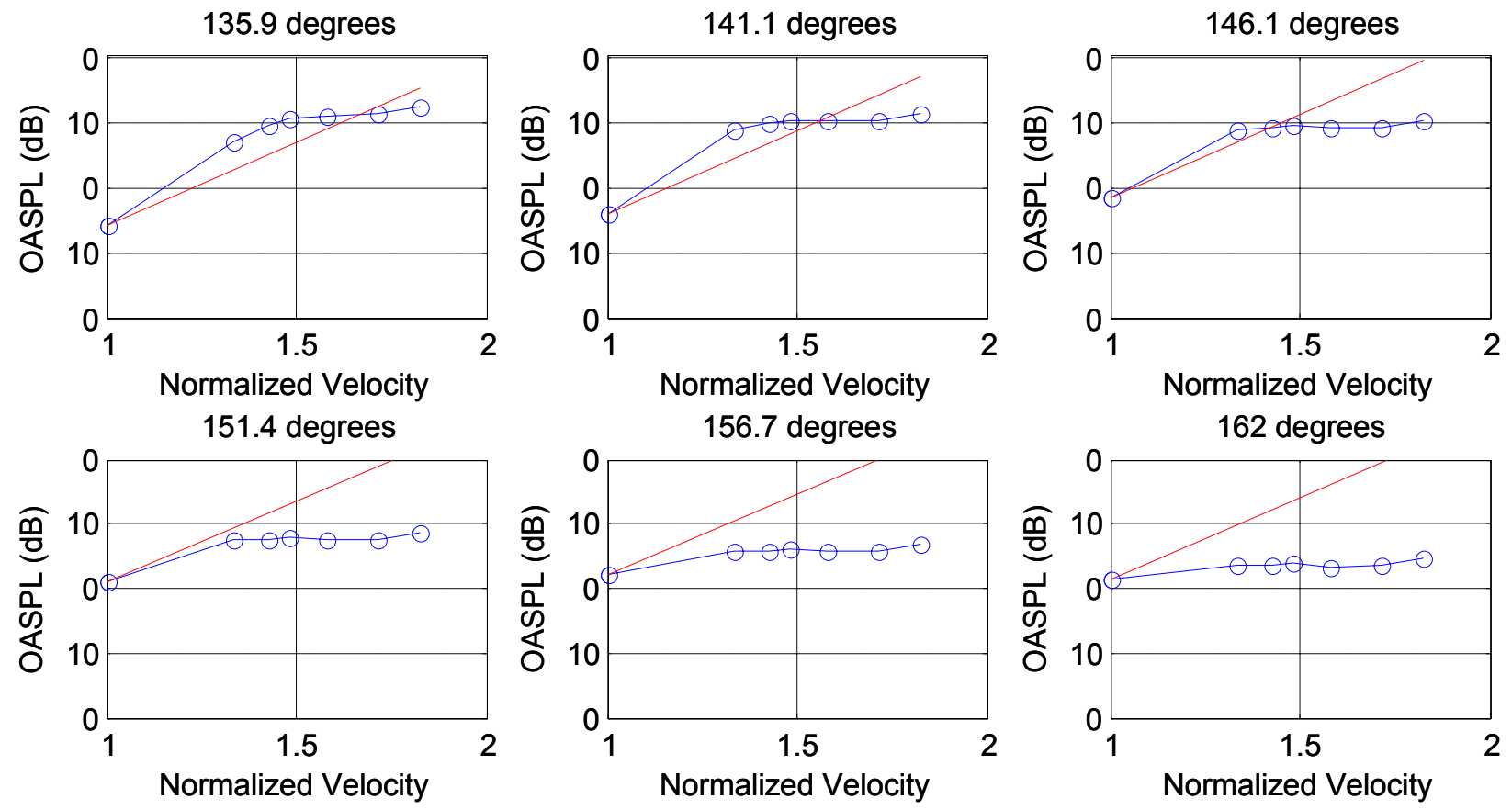

Figure 13. Overall sound pressure level vs. jet velocity for range of directivity angles: $M=1.5, T r=1.76$; raw data provided by James Bridges from Brown and Bridges (Ref. 24).

\section{Impulsive Waveforms and Propagation Features}

Supersonic jet noise is known to exhibit impulsive waveforms varying from weak Mach waves to strong compressions referred to as "crackle" propagating in aft directions. The latter phenomena was first identified and reported in 1975 by Ffowcs Williams (Ref. 28) at engine scale while Schlinker (Ref. 29) and Laufer, Schlinker, \& Kaplan (Ref. 30) identified the same phenomena in laboratory experiments. The current study seeks to track the statistical characteristic between the near field array and the far field microphone stations with the objective of determining the degree of non-linear propagation. For this purpose, times series of the near field as well as far field microphones were analyzed. The microphone stations are shown schematically in Figure 14 with measurements located at a minimum of 40 jet diameters.

Various authors have suggested that the impulsive character of the time series is due to non-linear wave propagation during which shock like impulsive waveform steepens increasing high frequency content. This conclusion was based on the skewness levels increasing with distance from the jet and observations of energy transfer from the central part of the spectrum to the high frequency end in prior studies. To investigate the steppening characteristic, skewness (Sk) was calculated and annotated for each of the jet near field microphone time series in Figure 15. Skewness levels are Sk 0.25-0.3 between $\mathrm{X} / \mathrm{D}=3$ to 10 with the larger values residing at $\mathrm{X} / \mathrm{D}=5$. While these values do not approach the $\mathrm{Sk}=0.4$ demarcation value selected by Ffowcs Williams to indicate the presence of crackle, the values clearly indicate non-linear features already exists in the near field within 2 radial diameters of the jet centerline. Also, the finite skewness over the $\mathrm{X} / \mathrm{D}=3-10$ diameter measurement range suggests that impulsive signature generation occurs over a distributed region rather than a localized station. 


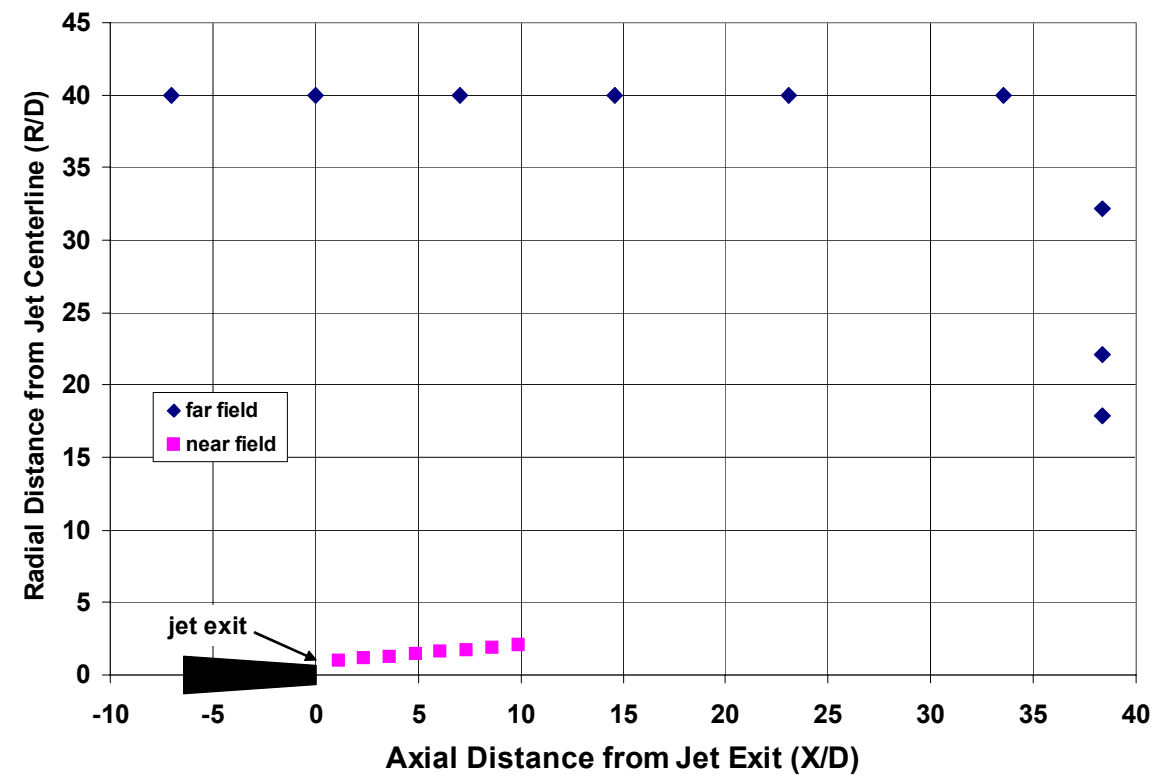

Figure 14. Near field and far field microphone stations.
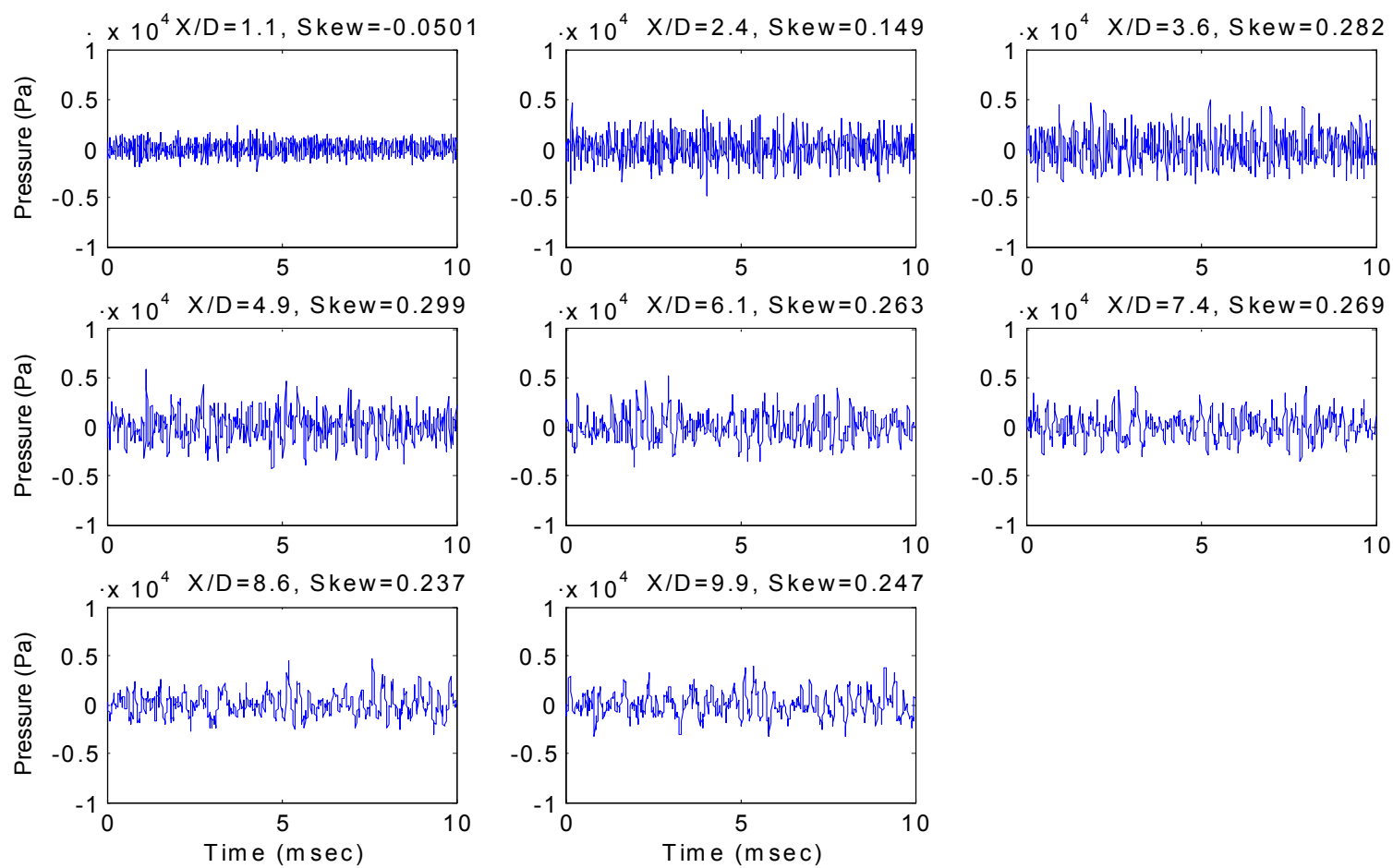

Figure 15. Near field pressure time series showing impulsive signature variation with microphone station and the corresponding skewness values: $M=1.5, \mathrm{Tr}=1.76$. 
To further track changes during propagation, skewness values were also calculated at the far field microphone stations shown in Figure 16. In this case, skewness values have doubled to $\mathrm{Sk} \sim 0.5-0.6$ with the pressure time series showing extreme impulsive signatures at the $130 \mathrm{deg}$ far field station. These measurements indicate strong non-linear propagation has occurred between microphones at 2 diameters from the jet centerline and the far field at $>40$ diameters. Also, the skewness peaks shift to upstream angles as expected with increasing convection speed due to heat addition.
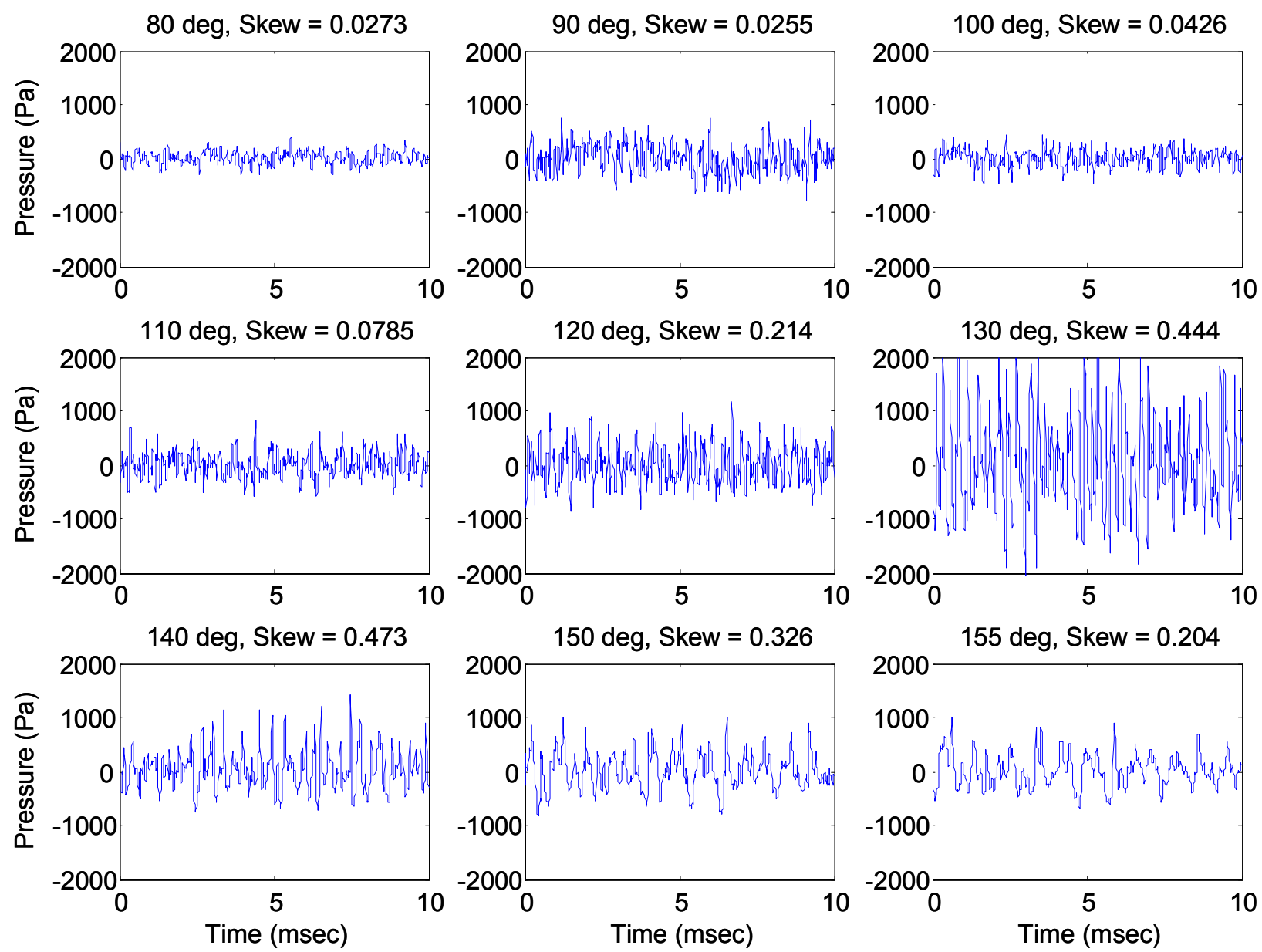

Figure 16. Far field pressure time series showing impulsive signature variation with directivity angle and the corresponding skewness values: $M=1.5, T r=1.76$.

A summary of the skewness dependence is given in Figure 17 for the far field microphone stations as a function jet velocity and directivity angle, theta. Heat addition was used to change jet velocity as indicated by the different static temperature ratios, annotated as $\mathrm{Tj} / \mathrm{Ta}$, for both subsonic and supersonic Mach numbers. Skewness is observed to increase dramatically at aft angles where Mach wave radiation is expected to increase in strength as jet velocity increases. The accentuated skewness in the narrow range of 130-140 deg follows expectations for Mach wave radiation fields. Further assessment of the propagation effects will be provided in future papers based on intermediate microphone station measurements to explicitly track propagation as a function of distance from the jet axis. 


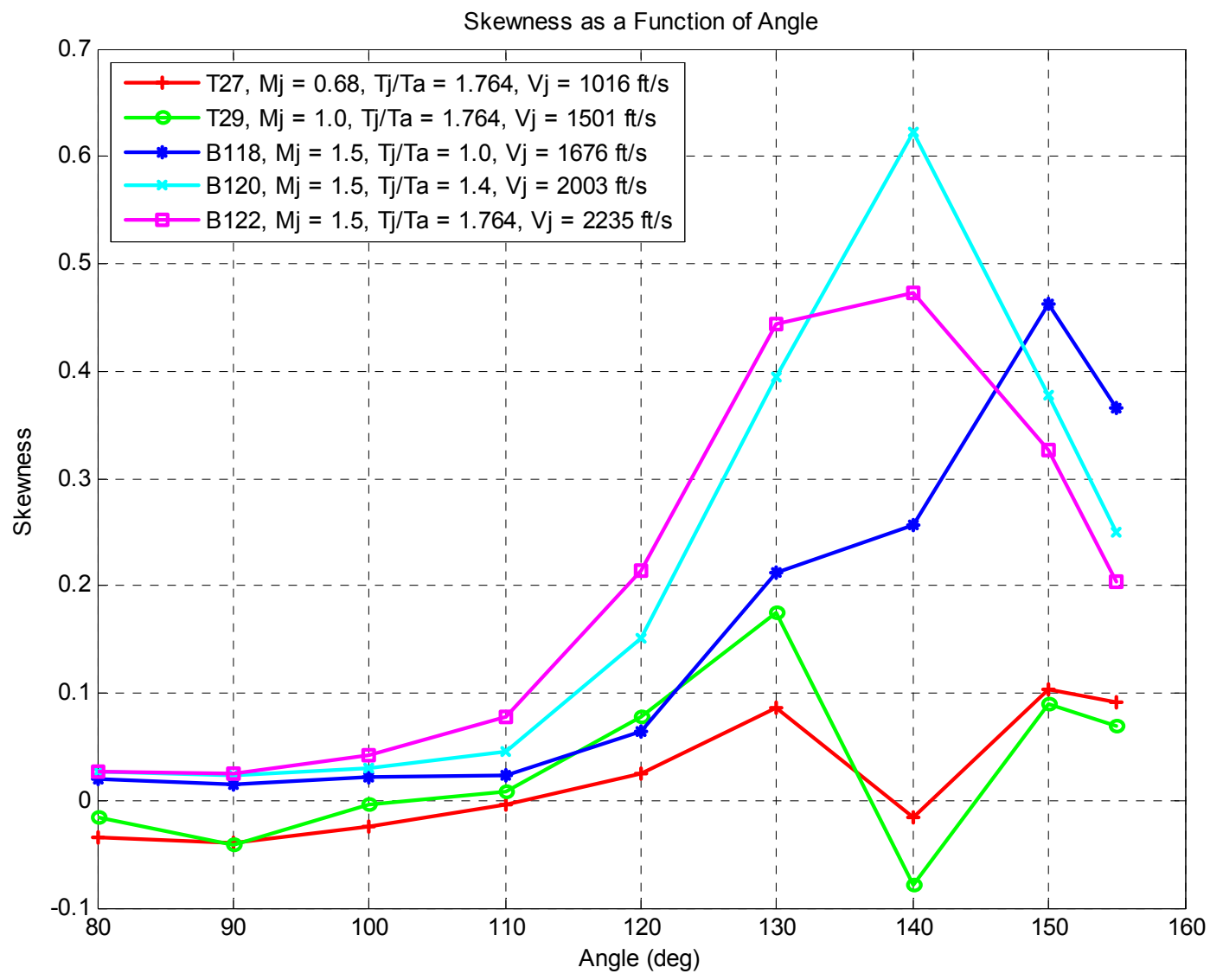

Figure 17. Skewness of far field acoustic pressure signals as a function of jet velocity and directivity angle.

\section{Summary}

A comprehensive program is in progress to: 1) develop fundamental knowledge of cause-and-effect between high speed jet exhaust large-scale structures and far field noise, and 2) acquire validation data for models/simulation methods characterizing large-scale turbulent structure noise generation with the long term goal of developing control strategies. While the concept of controlling the large scale/instability waves in jet flows has been envisioned for many years, its development and impact on noise reduction has been hindered by the lack of physics-based control strategies with prior studies focusing primarily on actuator devices. Therefore, a UTRC, Caltech, and TTC Technologies Team is developing unique modeling and experimental diagnostic capabilities and data bases suited for use in developing control strategies.

Details of a unique near field rotating phased array technique for extracting the spectral features and length scales of the large-scale turbulence noise sources have been described here. Laboratory scale high speed jet experiments providing source mechanism and validation data bases are presented for Mach numbers ranging from $\mathrm{M}=0.68$ to 1.5 while static temperature ratios ranged from $\operatorname{Tr}=0.68$ to 2 .

Measured supersonic model scale directivity patterns displayed several distinct features: 1) a "beam" of acoustic energy which is narrower than subsonic radiation patterns and approximately $20 \mathrm{deg}$. forward of the subsonic directivity peak, 2) a broader spectrum at the peak directivity angle compared to subsonic conditions, and 3) rapid sound pressure level changes with directivity angle for fixed frequencies. These features are similar to the full scale engine characteristics documented in a prior paper confirming the applicability of the model scale data base. 
OASPL variation with velocity in the aft direction was found to follow approximately $\mathrm{V}^{8}$ at angles near the directivity pattern peak (130-140 deg) similar to trends observed at engine scale. However, the velocity dependence becomes almost invariant at further aft angles possibly due to density effects which again follow engine scale results.

Near field array measurements identified acoustic waveforms with skewness values of approximately $\mathrm{Sk}=0.25$ indicating mild non-linearities already occur within two diameters of the jet centerline. Skewness values approach $\mathrm{Sk}=0.5-0.6$ at the peak directivity angle of $130 \mathrm{deg}$ for propagation distances $>40$ diameters from the jet exit indicating strong non-linear distortion occurs during propagation to the acoustic far field. Also, the skewness peaks move to upstream angles as expected with increasing convection speed due to heat addition. These trends are similar to results observed at engine scale.

Results reported here represent interim work with experiments and analysis continuing in the future.

\section{Acknowledgements}

The authors gratefully acknowledge the support of NAVAIR and TTC Technologies Inc., the SBIR partner in this work.

\section{References}

1. Schlinker, R. H., Liljenberg, S. A., Polak, D.R., Post, K.A., Chipman, C. T., and Stern, A. M., "Supersonic Jet Noise Source Characteristics and Propagation: Engine and Model Scale," AIAA Paper 2007-3623, $14^{\text {th }}$ AIAA/CEAS Aeroacoustics Conference, Rome, Italy, May 2007.

2. Mollo-Christensen, E., "Jet Noise and Shear Layer Instability Seen from an Experimenter's Viewpoint", American Society of Mechanical Engineers Journal of Applied Mechanics, Vol. 89, pp. 1-7, 1967.

3. Siddon, T.E., "Noise Source Diagnostics Using Causality Correlations", AGARD CP 131, Noise Mechanisms, pp. 7-1:7-13, 1973.

4. Seiner, J.M. and Reethof, G., "On the Distribution of Source Coherency in Subsonic Jets", AIAA paper 74-4.

5. Armstrong, R.R., Michalke, A. and Fuchs, H.V., "Coherent Structures in Jet Turbulence and Noise", AIAA J., Vol. 15(7), pp. 1011-1017, 1977.

6. $\quad$ Bridges, J.E. \& Wernet, M.P. "Turbulence Measurements of Separate Flow Nozzles with Mixing Enhancement Features", Paper 2002-2484, $8^{\text {th }}$ AIAA/CEAS Aeroacoustics Conference, Breckenridge, CO, June 2002.

7. Michalke, A., "On the Effect of Spatial Source Coherence on the Radiation of Jet Noise", J. Sound and Vibration, Vol. 55(3), pp. 377-394, 1977.

8. Fuchs, H.V., "Space Correlations of the Fluctuating Pressure in Subsonic Turbulent Jets", J. Sound and Vibration, Vol. 23, pp. 77-99, 1972.

9. Panda, J., Seasholtz, R.G., and Elam, K.A., "Measurement of Correlation Between Low Density, Velocity and Density*Velocity ${ }^{2}$ with Far-Field Noise in High Speed Jets", 8th AIAA/CEAS Aeroacoustics Conference, June 17-19, Breckenridge, CO, AIAA 2002-2485, 2002.

10. Ukeiley, L.S. \& and Ponton, M.K., "On the Near Field Pressure of a Transonic Axisymmetric Jet", Intl. J. of Aeroacoustics, Vol. 3(1), pp. 43-66, 2004.

11. Suzuki, T., and Colonius, T., "Instability Waves in a Subsonic Round Jet Detected using a Near-Field Phased Microphone Array," J. Fluid Mech. 565:197. 2006.

12. Tinney, C.E., Jordan, P., Antoine, and G. \& Delville, J., "A study in the Near Pressure Field of Co-Axial Subsonic Jets," Paper 2006-2589, $12^{\text {th }}$ AIAA/CEAS Aeroacoustics Conference, Boston, USA, May 2006. 
13. Muller, F., Vuillot, F., Rahier, G., Casalis, and G., Piot, E., "Experimental and Numerical Investigation of the Near Field Pressure of a High Subsonic Hot Jet," Paper 2006-2535, $12^{\text {th }}$ AIAA/CEAS Aeroacoustics Conference, Boston, USA, May 2006.

14. Hileman, J., Thurow, B., and Samimy, M., "Acoustic Source Localization Using a 3-D Microphone Array in a Mach 1.3 Jet", Paper 2002-0366, 40th Aerospace Sciences Meeting \& Exhibit, Reno, NV, January 2002.

15. Hileman, J., Thurow, B. \& Samimy, M., "Large-Scale Structure Evolution and Sound Emission in High-Speed Jets: RealTime Visualization with Simultaneous Acoustic Measurements." J. Fluid Mech., Vol. 544, pp. 277-307, 2005.

16. Samimy, M., Kim, J.H., Kastner, J., and Adamovich, I., "Noise Mitigation in High Speed and High Reynolds Number Jets Using Plasma Actuators," Paper 2007-3622, AIAA/CEAS Aeroacoustics Conference, Boston, USA, May 2006.

17. Reba, R.A., Narayanan, S., Colonius, T., Suzuki, T., "Modeling Jet Noise from Organized Structures Using Near Field Hydrodynamic Pressure", Paper 2005-3093, $11^{\text {th }}$ AIAA/CEAS Aeroacoustics Conference, Monterey, CA, May 2005.

18. Reba. R, Simonich, J., Schlinker, R.H., "Measurement of Source Wave-Packets in High-Speed Jets and Connection to Far Field," Paper 2008-2891, $15^{\text {th }}$ AIAA/CEAS Aeroacoustics Conference, Vancouver, Canada, May 2007.

19. Ladeinde, F., Cai, X., Alabi, K., Reba, R., Schlinker, R.H., and Simonich, J., "On the Connection Between Near-Field and Far-field Solutions of High Speed Jet Noise," Paper 2008-510, 46th Aerospace Sciences Meeting \& Exhibit, Reno, NV, January 2008 .

20. Gudmundsson, K. and Colonius, T., "Spatial Stability Analysis of Chevron Jet Profiles," Paper 2007-3599, 13 $3^{\text {th }}$ AIAA/CEAS Aeroacoustics Conference, Rome, May 2007.

21. Simonich, J., Narayanan, S. and Schlinker, R.H., "Facility and Data Quality Issues for Scale-Model Jet Noise Testing," Paper 2003-1057, $9^{\text {th }}$ AIAA/CEAS Aeroacoustics Conference, Hilton Head, SC, May 2003.

22. Tanna, H.K., Dean P. D., Burrin, R. H., "The Generation and Radiation of Supersonic Jet Noise, Part III, Turbulent Mixing Noise Data," AFAPL-TR-76-65, 1976.

23. Tanna, H.K, Dean, P.D, Fisher, M.J, “The Influence of Temperature on Shock-Free Supersonic Jet Noise”, J. Sound Vib., Vol. 39(4), 1975, pp. 429-460.

24. Brown, C., Bridges, J., “Small Hot Jet Acoustic Rig Validation,” NASA TM -2006-213234.

25. Bass, H.E., Sutherland, L.C., Zuckerman, A.J., Blackstock, D.T. and D.M. Hester, "Atmospheric Absorption of Sound: Further Developments," J. Acoustic Soc. Am., Vol. 97, Part 1, pp. 680-683, January 1995 and erratum J. Acoustic. Soc. Am., Vol. 99, Part 2, p. 1259, February 1996.

26. Gas Turbine Jet Exhaust Noise Prediction, Technical Report ARP876, Society of Automotive Engineers, Inc., November 1985.

27. Tam, C.K.W., Viswanathan, K., Ahuja, K.K., and Panda, J., "The Sources of Jet Noise: Experimental Evidence," Paper 2007-3641, 14 ${ }^{\text {th }}$ AIAA/CEAS Aeroacoustics Conference, Rome, Italy, May 2007.

28. Ffowcs-Williams, J.E., Simson, J., and Virchis, V.J., "Crackle: an Annoying Component of Jet Noise", Journal of Fluid Mechanics, Vol. 71, 1975, pp. 251-271.

29. Schlinker, R.H., Supersonic Jet Noise Experiments, PhD Thesis, Department of Aerospace Engineering, University of Southern California, 1975.

30. Laufer, J., Schlinker, R. H., and Kaplan, R. E., "Experiments on Supersonic Jet Noise”, AIAA Journal, Vol. 14, No. 4, April 1976, pp 489-497. 